\title{
Blocking of the Polaron Effect and Spin-Split Cyclotron Resonance in a Two-Dimensional Electron Gas
}

\author{
X. G. Wu \\ National Laboratory for Superlattice and Microstructure, Institute of Semiconductors, Chinese Academy of Sciences, \\ Beijing 100083, China \\ F. M. Peeters \\ Department of Physics, University of Antwerp (UIA), B-2610 Antwerp, Belgium \\ Y. J. Wang \\ National High Magnetic Field Laboratory at Florida State University, Tallahassee, Florida 32310 \\ B. D. McCombe \\ Department of Physics, State University of New York at Buffalo, Buffalo, New York 14260
}

(Received 14 December 1999)

\begin{abstract}
Cyclotron resonance (CR) of high density GaAs quantum wells exhibits well-resolved spin splitting above the LO-phonon frequency. The spin-up and spin-down CR frequencies are reversed relative to the order expected from simple band nonparabolicity. We demonstrate that this is a consequence of the blocking of the polaron interaction which is a sensitive function of the filling of the Landau levels.
\end{abstract}

PACS numbers: 71.38. $+\mathrm{i}$, 72.10.Di, 78.20.Ls

In a semiconductor with a nonzero effective Landé factor and a nonparabolic conduction band, e.g., GaAs, one expects to see spin splitting of the cyclotron resonance (CR) transitions when more than one spin state is occupied by electrons, as electrons with different spin orientations have different cyclotron frequencies. This is commonly observed in bulk GaAs samples of high quality. However, for quasi-two-dimensional electron systems (2DES) formed in GaAs-AlGaAs heterostructures and quantum wells, this spin splitting was not observed [1-3] until recently [4,5].

In earlier experiments, with sample electron densities of the order of $10^{11} \mathrm{~cm}^{-2}$, only a single CR peak was resolved, though samples had very high electron mobility [1-3]. Spin-split CR was observed only in samples with very low electron densities, of the order of $10^{10} \mathrm{~cm}^{-2}[4,5]$ for which the electron-electron interaction plays a very important role. As the electron density increases above $5 \times$ $10^{10} \mathrm{~cm}^{-2}$, the two CR peaks merge into a single resonance, and this has been attributed to electron-electron interaction [4-7]. As the CR should not be affected by the electron-electron interaction in a translationally invariant system [8] invoking the electron-electron interaction requires breaking the translational invariance, e.g., by conduction band nonparabolicity, electron-impurity scattering, or electron-phonon interaction [6,7,9-12].

In far infrared magneto-optical studies of electron $\mathrm{CR}$ in heavily doped multiple-quantum-well (MQW) GaAs/AlGaAs samples at high magnetic fields we have observed an unusual, very large splitting of the CR above the longitudinal optical phonon energy of GaAs. The splitting occurs in samples having electron densities more than an order of magnitude higher than in any previous observation, and it vanishes when the electron density is further increased or decreased. The two lines are observed only when the spin-up state of the lowest Landau level is fully occupied and the spin-down state is partially occupied. We attribute these two lines to spin-split CR. The unusually large splitting and a reversal of the order of the strength of the two lines (relative to that expected from band nonparabolicity) results from Pauli-principle blocking of the resonant polaron interaction. For the highest density sample the higher frequency CR line occurs below the unperturbed (only band nonparabolicity) result, in contrast to all previous measurements. At low magnetic fields, i.e., below the reststrahlen region, only a single CR line can be identified, consistent with previous experiments [1-3].

We substantiate the above assertions through detailed calculations which clearly show that the observations result from Pauli-principle blocking of the resonant electronoptical phonon interaction for one of the spin states due to Landau-level filling. This leads to a reversal of the frequency ordering of the CR lines as compared to the one expected from nonparabolicity: the spin-up CR becomes the lower energy line. These calculations also show that for very high density samples the higher frequency $\mathrm{CR}$ is depressed below the unperturbed (by electron-optical phonon interaction) calculated $\mathrm{CR}$ position due to a resonant three level polaron interaction with the first excited subband.

The CR measurements were performed on GaAs/ $\mathrm{Al}_{0.3} \mathrm{Ga}_{0.7} \mathrm{As} \mathrm{MQW}$ structures grown by molecular-beam epitaxy. In all cases the QW widths are $24 \mathrm{~nm}$ and the barrier widths are $48 \mathrm{~nm}$. The barriers are centrally doped with $\mathrm{Si}$ donors which leads to electron densities in the wells of $3 \times 10^{11}, 7.5 \times 10^{11}$, and $12 \times 10^{11} \mathrm{~cm}^{-2}$. The far infrared transmission measurements were carried 
out at $4.2 \mathrm{~K}$ in magnetic fields up to $30 \mathrm{~T}$ at the National High Magnetic Field Laboratory.

In Fig. 1, the positions of the transmission minima are plotted versus magnetic field as open circles for the three samples. The full width at half strength of the transmission minima is $9-13 \mathrm{~cm}^{-1}$, much larger than the inherent linewidth because of overabsorption. As shown in the figure, at low magnetic fields, i.e., below the GaAs reststrahlen region, only one CR peak is resolved for all samples. At high magnetic fields, above the electron LO-phonon resonance $\left(\lambda_{\mathrm{LO}}^{-1}=296.4 \mathrm{~cm}^{-1}\right)$, two clearly resolved CR peaks are observed for the sample with intermediate electron density [Fig. 1(b), $\left.n_{e}=7.5 \times 10^{11} \mathrm{~cm}^{-2}\right]$. The intensity of the lower frequency branch is about 3-4 times larger than that of the higher frequency branch. In contrast, for samples of lower [Fig. 1(a), $n_{e}=3 \times 10^{11} \mathrm{~cm}^{-2}$ ] and higher [Fig. 1(c), $n_{e}=12 \times 10^{11} \mathrm{~cm}^{-2}$ ] electron densities, only a single $\mathrm{CR}$ peak can be identified. For the region between 18 and $24 \mathrm{~T}$ there is little infrared light passing through the samples because of the strong absorption of the GaAs reststrahlen band. The width of the transmission minima increases with magnetic field above the reststrahlen region.

Previous experimental and theoretical studies have shown that, in analyzing the CR of quasi-2DES in GaAs-AlGaAs heterostructures and quantum wells, it is essential to include the conduction band nonparabolicity, the electron-phonon interaction, and the electron-electron interaction [1-3,11-14]. Our model includes these important ingredients from the beginning. In order to make a quantitative comparison with experiment, the electron subband structure is calculated self-consistently [15].

The polaron correction to the CR frequency is calculated by the well-established memory function approach [14,16-18]. In the memory function method, the dynamical conductivity $\sigma(\omega)=\sigma_{x x}(\omega)+i \sigma_{x y}(\omega)$, which is

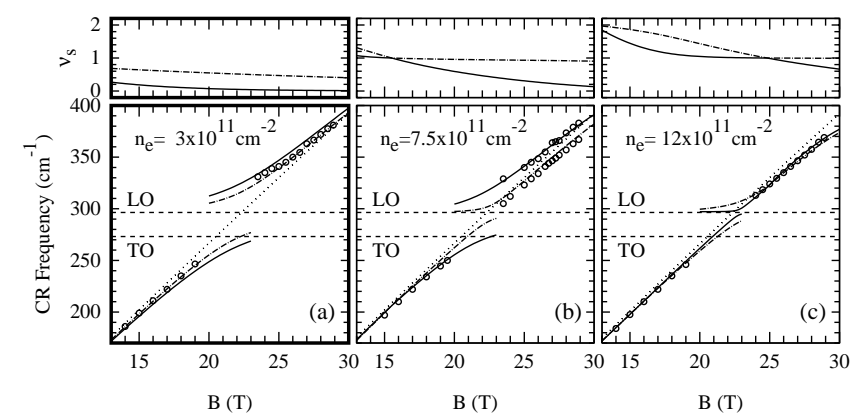

FIG. 1. The lower panels show the measured CR frequency versus the magnetic field (open circles) for three samples of different electron densities. The calculated CR frequency is shown as solid (dash-dotted) curves for the spin-down, $s=-1$ (up, $s=1$ ) transitions, including the conduction band nonparabolicity and the electron bulk LO-phonon coupling. The dotted lines show the CR frequency with band nonparabolicity only. The corresponding upper panels show the Landau-level occupancy of the spin-down (solid curve) and spin-up (dash-dotted curve) electrons, respectively. related to the physical quantity measured in a CR experiment, can be written as [16-18]

$$
\sigma(\omega)=\sum_{s} \frac{i\left\langle N_{s}\right\rangle e^{2} / m_{b}}{\omega-\omega_{c}-M_{s}(\omega)},
$$

where the subscript $s= \pm 1$ labels the electron spin. $\left\langle N_{s}\right\rangle$ is the averaged number of electrons with spin $s, m_{b}$ the electron band mass, and $\omega_{c}=e B / m_{b} c$ the unperturbed CR frequency. We used the well-known value $m_{b} / m_{0}=$ 0.0665 and $\alpha=0.068$ for the electron-phonon coupling strength. $M_{s}(\omega)$ is the memory function due to electron bulk LO-phonon coupling. Treating the electron-phonon interaction as a perturbation, $M_{s}(\omega)$ can be written as $M_{s}(\omega)=(1 / \omega) \int_{0}^{\infty} d t\left(1-e^{i \omega t}\right) \operatorname{Im} F_{s}(t)$, with

$$
\begin{aligned}
F_{s}(t)= & \sum_{\mathbf{k}} \frac{k^{2}\left|V_{k}\right|^{2}}{\left\langle N_{s}\right\rangle m_{b} \hbar} \\
& \times\left\{n\left(\omega_{\mathrm{LO}}\right)\left\langle n_{-\mathbf{k} s}(0) n_{\mathbf{k} s}(t)\right\rangle\right. \\
& \left.\quad-\left[1+n\left(\omega_{\mathrm{LO}}\right)\right]\left\langle n_{\mathbf{k} s}(t) n_{-\mathbf{k} s}(0)\right\rangle\right\} e^{-i \omega_{\mathrm{LO}} t}
\end{aligned}
$$

where $n\left(\omega_{\mathrm{LO}}\right)$ is the number of phonons, $\omega_{\mathrm{LO}}$ the LO-phonon frequency, and $n_{\mathbf{k} s}=\int n_{s}(\mathbf{r}) e^{i \mathbf{k} \cdot \mathbf{r}} d \mathbf{r}$ with $n_{s}$ the density of electrons with spin $s$. The CR frequency $\left(\omega_{s}\right)$ corrected for electron-phonon coupling is obtained by solving equation $\omega_{s}-\omega_{c}-M_{s}\left(\omega_{s}\right)=0$, for $s= \pm 1$. The effect of conduction band nonparabolicity is included in a local parabolic band approximation, in which a two-band Kane model is employed [3,14,18]. It is clear that there are two CR peaks in general, and the size of the splitting is determined by the memory function. The electron-electron interaction can couple these two CR transitions [6,7,19-21] producing a single mode or two mixed modes.

In the lower panels of Fig. 1, the calculated CR frequency is shown as solid (dash-dotted) curves for the spin down $s=-1$ (spin up: $s=1$ ) transitions. To appreciate the importance of electron-phonon coupling, the calculated spin-split CR frequency with only band nonparabolicity included is shown by the dotted lines in Fig. 1 (there are two dotted lines, but as the difference is small, they are hardly distinguishable in the figure). It is clear that the electron-phonon interaction produces large corrections to the CR frequency not only in the resonant region $\left(\hbar \omega_{c} \sim \hbar \omega_{\mathrm{LO}}\right)$, but also in the off-resonant region. We included the Pauli-principle blocking effect which is due to Fermi-Dirac statistics and is known to reduce the effective electron-phonon coupling strength $[3,14,18]$. The screening of the electron-phonon coupling due to electron-electron interaction is found to produce a negligible correction to the $\mathrm{CR}$ frequency (about $1 \mathrm{~cm}^{-1}$ ). The Pauli-principle blocking leads to a reduction of the resonant polaron splitting near the LO-phonon energy for CR originating in a given Landau-level, spin state $\left(N_{s}\right)$ for densities such that the state is near full occupancy; this results in a concomitant increase in the spin splitting when the other spin state is only partially occupied. The 
single-particle electron-phonon interaction is the same for both spin configurations, but the many-particle effects due to the different occupancy of the two spin states lead to (very) different polaron corrections to the spin-up and spin-down CR transitions for the upper branch in the resonant polaron region.

The upper panels in Fig. 1 show the Landau-level occupancy of electrons with spin $s, \nu_{s}=\left(\left\langle N_{s}\right\rangle / A\right)(h c / e B)$, where $A$ is the area of the 2DES, and the spin-down (up) values are given by the solid (dash-dotted) curve. Thus $\nu=\nu_{+1}+\nu_{-1}$ is the filling factor. When $\nu_{s}$ is near an integer value the polaron correction is almost absent, i.e., it is blocked. This is most clearly seen in the case of Fig. 1(b) where for $B>20 \mathrm{~T}$ the spin-up state (dashdotted curve) has $\nu_{s=1} \sim 1$. The corresponding $\mathrm{CR}$ has almost no electron-phonon correction while the spin-down state with $\nu_{s=-1} \ll 1$ is appreciably shifted by the electron-phonon interaction. As a result, above the reststrahlen region the spin-down state has a higher CR transition frequency than the spin-up state, opposite to the (normal) situation below the LO-phonon frequency. This now explains the observation that above the LO-phonon frequency for the $n_{e}=7.5 \times 10^{11} \mathrm{~cm}^{-2}$ sample the lowest frequency CR line is the strongest; it corresponds to the spin-up transition which has the highest occupation. Thus above the LO-phonon frequency the CR spin-up and spin-down transitions are reversed with respect to the order expected solely from band structure effects. The influence of the different corrections to the Landau levels and the CR energy is schematically illustrated in Fig. 2.

Another remarkable observation is that for the highest density sample [see Fig. 1(c)] the measured CR above the LO-phonon frequency lies below the unperturbed (only inclusion of band nonparabolicity) result (this is also apparent in the spin-up transition for the $n_{e}=7.5 \times 10^{11} \mathrm{~cm}^{-2}$ density sample) and that the theo-
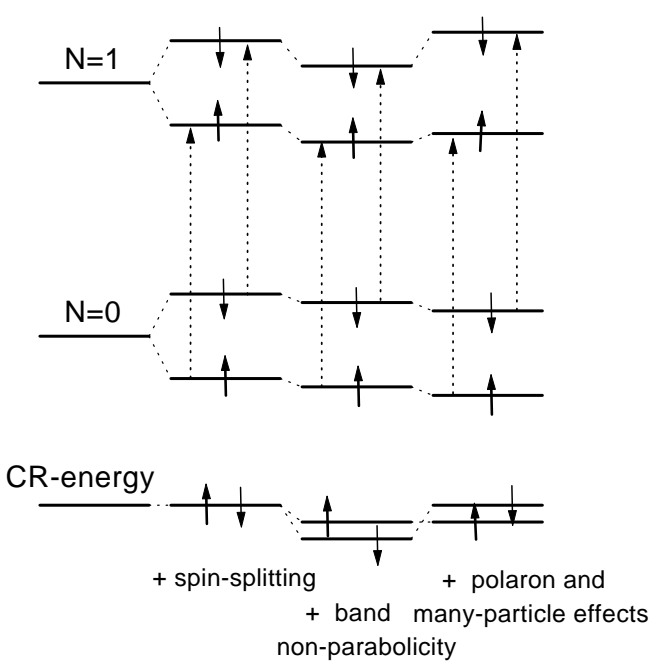

FIG. 2. Schematic view of the influence of the different effects on the position of the energy levels and the CR transition energy. The polaron correction is for the case of $\omega_{c}>\omega_{\mathrm{LO}}$. retical spin-up and spin-down curves cross each other. This can be understood only by incorporating higher subbands which lead to a three level resonant polaron interaction. As the electron density increases, the energy separation between the second and the first electric subband $\varepsilon_{2}-\varepsilon_{1}$ decreases [15]. At high magnetic fields, $\hbar \omega_{c}$ becomes comparable to $\hbar \omega_{\mathrm{LO}}+\varepsilon_{2}-\varepsilon_{1}\left[=462,428,402 \mathrm{~cm}^{-1}\right.$ for $n_{e}=(3,7.5,12) \times 10^{11} \mathrm{~cm}^{-2}$, respectively], and leads thus to an additional resonant contribution to the memory function which lowers the CR frequency. A resonant splitting similar to the one around the reststrahlen region will occur for $B>30 \mathrm{~T}$ and results in the crossing of the spin-up and spin-down CR frequencies in the highest density sample. This intersubband electron-phonon coupling, however, is weaker than the intrasubband one.

As is apparent from Fig. 1, the correction to the CR frequency due to electron-phonon interaction depends strongly on the electron density, i.e., the occupation of the Landau levels. This is more clearly demonstrated in Fig. 3. In the lower panels of Figs. 3(a) and 3(b), the
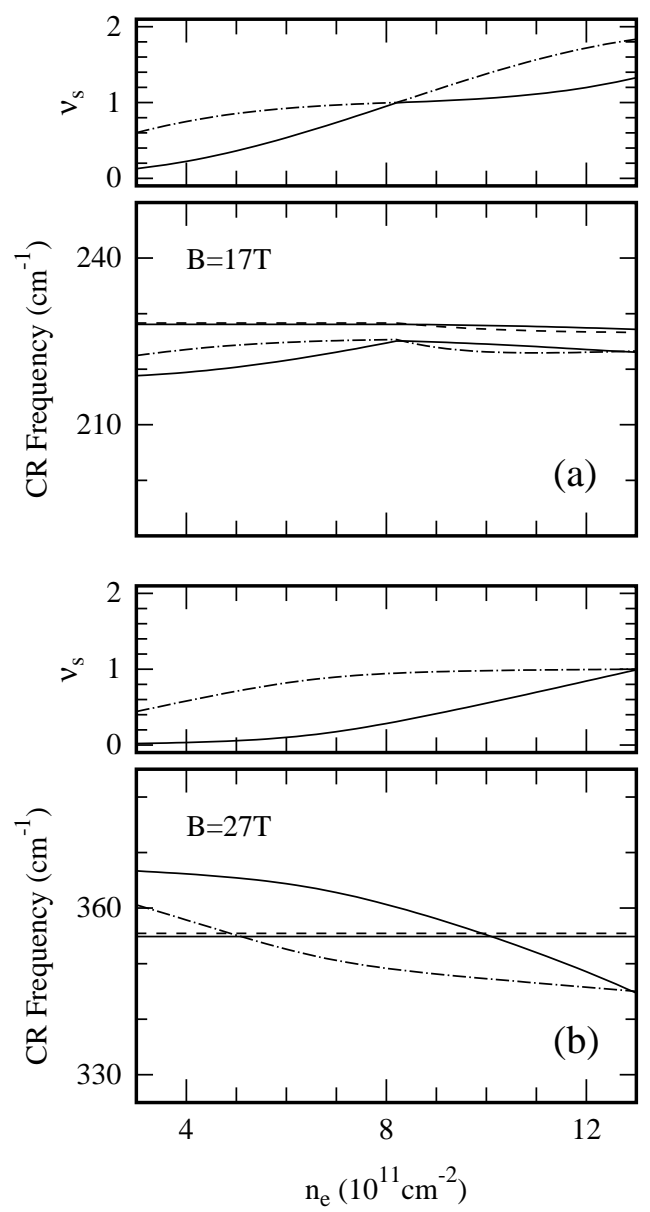

FIG. 3. (a) The calculated CR frequencies (the lower panel) and Landau-level occupancies (the upper panel) versus the electron density for a fixed magnetic field $B=17 \mathrm{~T}$. The solid (dash-dotted) curve corresponds to the spin-down, $s=-1$ (up, $s=1)$ transition. The thin solid and dashed curves show the CR frequency with only band nonparabolicity. (b) The same as (a), but for $B=27 \mathrm{~T}$. 
calculated CR frequency is shown versus electron density for fixed magnetic field: (a) $B=17 \mathrm{~T}$ and (b) $B=27 \mathrm{~T}$, respectively. In each corresponding upper panel, the electron Landau-level occupancy $\nu_{s}$ is shown. The solid and dash-dotted curves correspond to the spin-down $(s=-1)$ and spin-up $(s=1)$ transitions, respectively. For comparison, the CR frequency with only band nonparabolicity included is shown by the full (spin-down) and dashed (spin-up) thin lines. This figure nicely illustrates the above interpretation and the oscillatory dependence of the polaron correction to the spin splitting of the CR transition energy which is closely linked to the filling of the separate spin states. Above the LO-phonon frequency [Fig. 3(b)] the largest spin splitting is observed in the density range $(4-9) \times 10^{11} \mathrm{~cm}^{-2}$.

Note that in the experiment, due to the finite width of the CR lines, the spin splitting is not observed when the two spin-split lines are too close in energy. This is so below the reststrahlen region, and also above the reststrahlen region when the ratio between the occupancy of the two spin states is close to 1 . Another reason can be that the electron-electron interaction couples the two CR transitions which may lead to a single mode as was found in previous $\mathrm{CR}$ studies [5,6,19-21]. We considered the influence of electron-electron interaction phenomenologically along the lines of [19-21], which is equivalent to that used in $[5,6,11,12]$. In this approach, the spin-split CR calculated above is identified as the CR of two types of electrons with different effective masses $m_{s} / m_{b}=\omega_{c} / \omega_{s}$. Within the approximation of frequency independent electron-electron scattering time, the relaxation of the relative total momentum $\boldsymbol{\Pi}$ is governed by $d \boldsymbol{\Pi} / d t=M_{e}(\omega) \boldsymbol{\Pi}=\left(i \omega_{e}-1 / \tau_{e}\right) \boldsymbol{\Pi}$. This model has been well studied, where $\omega_{e}$ shifts the CR frequencies, while $\tau_{e}$ broadens the CR peaks [19-21]. Such a study indicated that we are in the weak electron-electron coupling regime with almost no mixing of the two modes. Therefore, we believe that the splitting is smeared out by the broadening of the CR peaks leading to the observation of a single CR peak in the case of small spin splitting. The effect of well thickness inhomogeneity should not affect our results much. These three samples do not have many wells: the most lightly doped sample has five wells, the intermediate one three wells, and the highest doped sample has two wells. The inhomogeneity of well thickness might contribute to the linewidth broadening of the CR, but nothing else.

In summary, we have observed an unusual splitting of electron CR in the region of the resonant polaron interaction at high magnetic fields for GaAs/AlGaAs MQW samples having high electron densities. The splitting is very large compared to $\mathrm{CR}$ spin splitting expected from band structure effects, and also entails a reversal of the frequency ordering of the strengths of the lines expected from band structure and occupancy of the spin states. This extraordinary behavior occurs only when one spin state of the lowest Landau level is essentially fully occupied and the other is partially occupied. A model without any fitting parameters gives an excellent account of the data, and demonstrates that this unusual behavior results from essentially complete Pauli-principle blocking of the resonant polaron interaction for one of the spin states.

X.G.W. was partly supported by the NSF of China, F. M.P. by the Flemish Science Foundation (FWO-Vl), B. D. M. was supported, in part, by NSF DMR9722625, and Y.J.W. was supported by the NHMFL. This work was supported by the Bilateral Flanders-China agreement, FWO-Vl and IUAP-VI. Discussions with R. Nicholas are gratefully acknowledged.

[1] J. G. Michels, S. Hill, R. J. Warburton, G. M. Summers, P. Gee, J. Singleton, R. J. Nicholas, C. T. Foxon, and J. J. Harris, Surf. Sci. 305, 33 (1993).

[2] E. Batke, K. Bollweg, U. Merkt, C. M. Hu, K. Köhler, and P. Ganser, Phys. Rev. B 48, 8761 (1993).

[3] F. M. Peeters, X. G. Wu, J. T. Devreese, C. J. G. M. Langerak, J. Singleton, D. J. Barnes, and R.J. Nicholas, Phys. Rev. B 45, 4296 (1992).

[4] G. M. Summers, R. J. Warburton, J. G. Michels, R. J. Nicholas, J. J. Harris, and C. T. Foxon, Phys. Rev. Lett. 70, 2150 (1993).

[5] C. M. Hu, T. Friedrich, E. Batke, K. Köhler, and P. Ganser, Phys. Rev. B 52, 12090 (1995).

[6] N. R. Cooper and J. T. Chalker, Phys. Rev. Lett. 72, 2057 (1994).

[7] U. Merkt, Phys. Rev. Lett. 76, 1134 (1996).

[8] W. Kohn, Phys. Rev. 123, 1242 (1961).

[9] A. H. MacDonald and C. Kallin, Phys. Rev. B 40, 5795 (1989).

[10] D. Antoniou and A.H. MacDonald, Phys. Rev. B 46, 15225 (1992).

[11] C. M. Hu, E. Batke, K. Köhler, and P. Ganser, Phys. Rev. Lett. 75, 918 (1995).

[12] C. M. Hu, E. Batke, K. Köhler, and P. Ganser, Phys. Rev. Lett. 76, 1904 (1996).

[13] Y. J. Wang, Z.X. Jiang, B.D. McCombe, F. M. Peeters, X. G. Wu, G. Q. Hai, T. J. Eustis, and W. Schaff, Physica (Amsterdam) 256B-258B, 292 (1998).

[14] Y. J. Wang, H. A. Nickel, B. D. McCombe, F. M. Peeters, J. M. Shi, G. Q. Hai, X. G. Wu, T. J. Eustis, and W. Schaff, Phys. Rev. Lett. 79, 3226 (1997).

[15] T. Ando, A. B. Fowler, and F. Stern, Rev. Mod. Phys. 54, 437 (1982).

[16] W. Götze and P. Wölfe, Phys. Rev. B 6, 1226 (1972).

[17] C. S. Ting, S. C. Ying, and J. J. Quinn, Phys. Rev. B 16, 5394 (1977).

[18] X. G. Wu, F. M. Peeters, and J. T. Devreese, Phys. Rev. B 36, 9760 (1987).

[19] J. Appel and A. W. Overhauser, Phys. Rev. B 18, 758 (1978).

[20] C. S. Ting, A. K. Ganguly, and W. Y. Lai, Phys. Rev. B 24, 3371 (1981).

[21] B. E. Cole, F. M. Peeters, A. Ardavan, S. O. Hill, J. Singleton, W. Batty, J. M. Chamberlain, A. Polisskii, M. Henini, and T. Cheng, J. Phys. Condens. Matter 9, 3163 (1997). 